\title{
Pour une refonte des programmes pour les travailleurs migrants temporaires
}

Mimi Zou

\section{(2) OpenEdition}

1 Journals

Édition électronique

URL : https://journals.openedition.org/rdctss/1972

DOI : $10.4000 /$ rdctss. 1972

ISSN : 2262-9815

Éditeur

Centre de droit comparé du travail et de la sécurité sociale

\section{Édition imprimée}

Date de publication : 1 avril 2018

Pagination : 18-31

ISSN : 2117-4350

\section{Référence électronique}

Mimi Zou, "Pour une refonte des programmes pour les travailleurs migrants temporaires », Revue de droit comparé du travail et de la sécurité sociale [En ligne], 1 | 2018, mis en ligne le 01 novembre 2021, consulté le 12 novembre 2021. URL : http://journals.openedition.org/rdctss/1972 ; DOI : https:// doi.org/10.4000/rdctss. 1972

\section{(c) (†) $९$}

Revue de droit comparé du travail et de la sécurité sociale est mise à disposition selon les termes de la Licence Creative Commons Attribution - Pas d'Utilisation Commerciale - Pas de Modification 4.0 International. 


\title{
POUR UNE REFONTE DES PROGRAMMES POUR LES TRAVAILLEURS MIGRANTS TEMPORAIRES*
}

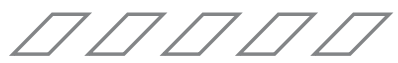

\section{RÉSUMÉ}

Au début du $21^{\text {ème }}$ siècle, les TMWP (programmes pour les travailleurs migrants temporaires) ont été créés et se sont développés dans de nombreux États développés, libéraux et industrialisés. Cet article examine les restrictions les plus problématiques en matière d'emploi et de droits sociaux des migrants, dans des pays d'accueil démocratiques libéraux. En examinant et en critiquant un certain nombre de propositions en vue de la refonte des TMWP, cet article de s'appuyer sur le compromis «Exit, Voice » dans les relations de travail des migrants pour envisager la refonte des principales caractéristiques des TMWP actuellement en cours.

MOTS CLÉS: Main-d'œuvre migrante, migration temporaire, droit de l'immigration et du travail, emploi et droits sociaux, travailleurs immigrés.

\begin{abstract}
At the beginning of the $21^{\text {st }}$ century, (TMWPs) have emerged and expanded in numerous advanced industrialised, liberal states. This paper probes into the most problematic restrictions of migrants' employment and social rights under such schemes in liberal democratic host states. In reviewing and critiquing a number of notable proposals for redesigning TMWPs, this paper puts forward the discourse of 'exit and voice' in migrants' work relations as a normative foundation for redesigning the key features of current TMWPs.
\end{abstract}

KEY WORDS: Labour Migration Programmes, Migrant Workers' Rights, Temporary Labour Migration, Immigration and Labour Laws, Employment and Social Rights.

* La version originale de cet article en anglais est parue dans Lavoro e Diritto, vol. 1. 2017, pp. 91-109. 
admission au séjour de travailleurs migrants temporaires est devenue un moyen de plus en plus répandu pour accéder au marché du travail dans les pays industrialisés, notamment dans ceux qui ont traditionnellement mis l'accent sur l'installation permanente des travailleurs migrants ${ }^{1}$. En cherchant à différencier les TMWP contemporains des systèmes de travailleurs "invités » d'après-guerre, "moins humains ", les défenseurs de ces nouveaux instruments soutiennent que "conçus avec soin » ils peuvent constituer un instrument politique permettant de gagner sur trois tableaux ${ }^{2}$.

Ils soutiennent premièrement que les TMWP peuvent être un élément de réponse aux besoins de l'économie et du marché du travail dans les pays d'accueil, tout en apaisant les inquiétudes politiques nationales concernant les migrations permanentes. Deuxièmement, les pays d'origine peuvent bénéficier des envois de fonds et des transferts de compétences de leurs ressortissants, tout en atténuant le problème de la «fuite des cerveaux ». Troisièmement, pour les migrants et leurs familles, les TMWP donnent accès à des opportunités d'emploi à l'étranger par des voies légales. D'un autre côté, les critiques ont mis en évidence le caractère intrinsèquement abusif de ces mêmes instruments qui imposent des restrictions en matière d'emploi et de droits sociaux des migrants et qui fournissent aux employeurs des moyens de contrôle supplémentaires sur le lieu de travail et au-delà ${ }^{3}$.

Les différentes positions adoptées dans ce débat soulèvent la question de la posture morale à adopter vis-à-vis de migrants qui ne sont pas citoyens des pays d'accueil. Cette contribution commence par critiquer une proposition relativement influente en faveur d'un arbitrage entre le "nombre de migrants et les droits des migrants ", qui suggère qu'il existe un compromis politique possible entre l'ouverture de la politique d'immigration d'un pays d'accueil d'une part et les droits accordés aux migrants admis d'autre part (I)4. Nous verrons par la suite quelques propositions importantes pour « améliorer » les TMWP qui ont été avancées par la doctrine, notamment dans l'idée d'accorder aux migrants un ensemble restreint de droits «fondamentaux » (II). La dernière partie de cette contribution

1 Commission mondiale sur les migrations internationales (GCIM), La migration dans un monde interconnecté: nouvelles orientations pour l'action, Suisse, GCIM, 2005, p. 16.

2 M. Ruhs, « Le potentiel des programmes de migration temporaire dans la future politique migratoire internationale », Revue internationale du travail, $n^{\circ} 145,2006$, p. 7.

3 S. Castles, «Guestworkers in Europe: A Resurrection? »International Migration Review, vol. 40, 2006, p. 741 ; C. Dauvergne et S. Marsden, "The Ideology of Temporary Labour Migration in the Post-Global Era », Citizenship Studies, vol. 18, 2014, p. 224 ; B. Anderson, "Migration, immigration controls and the fashioning of precarious workers", Work, Employment and Society, vol. 24, 2010, p. 300 ; J. Fudge, "Precarious Migrant Status and Precarious Employment: The Paradox of International Rights for Migrant Workers", Comparative Labour Law and Policy Journal, vol. 34, n 1 , 2012, p. 96.

4 M. Ruhs, The Price of Rights: Regulating International Labor Migration, Princeton University Press, 2013, p. 173. 
(III) présente des idées sur la réforme possible des aspects les plus problématiques des TMWP, consistant à élargir les possibilités de mettre un terme à leur relation de travail et de s'exprimer et plus largement de pouvoir faire cela dans le cadre de leur projet migratoire.

\section{I - « NUMBERS VERSUS RIGHTS »?}

Un des aspects majeurs des revendications «triplement gagnantes » en matière de TMWP concerne les prétendus avantages découlant de la possibilité offerte aux migrants d'accéder à l'emploi dans des pays d'accueil à revenu élevé. De tels arguments présupposent généralement que la plupart des migrants temporaires n'ont souvent pas l'intention de résider à long terme dans le pays d'accueil, mais souhaitent simplement avoir accès à des opportunités économiques afin «d'améliorer leurs conditions de vie » dans la perspective du retour dans leur pays ${ }^{5}$.

Les défenseurs des TMWP contemporains affirment que de tels systèmes élargissent les canaux légaux de la migration mondiale économique face à de puissants facteurs d'incitation et d'attraction. Sans cela, ils estiment que les migrants peuvent être poussés vers des secteurs informels et moins réglementés du marché du travail sur lesquels ils sont plus vulnérables et sujets à exploitation 6 .

Comme Pevnick le fait remarquer, il est peu probable que les travailleurs étrangers préfèrent un système avec des droits légaux complets excepté, un très petit nombre d'entre eux (poussant ainsi de nombreuses personnes vers l'immigration clandestine) plutôt qu'un programme relativement humain pour les travailleurs invités, permettant l'entrée $d^{\prime} u n$ nombre suffisant de travailleurs pour satisfaire la demande en main-d'œuvre ${ }^{7}$. Les TMWP sont donc considérés comme une politique de " second choix ", face à l'alternative « politiquement irréalisable » d'accorder la résidence permanente à tous les migrants.

Les arguments en faveur de l'extension des TMWP accordent un poids considérable à la proposition d'un compromis entre accueillir « en nombre de migrants et accorder des droits aux migrants". Dans son analyse des TMWP dans plus de 50 pays à revenu élevé, Ruhs estime que plus la politique d'admission est " ouverte »(en nombre de migrants), plus l'ensemble des droits accordés aux migrants est restreint, et inversement. II soutient que les droits des migrants ont non seulement une valeur intrinsèque, comme le soulignent les approches axées sur les droits de l'homme, mais ces droits jouent également un rôle déterminant sur les effets des migrations économiques pour les pays d'accueil, les États d'origine et les migrants eux-mêmes.

Pour Ruhs, les droits accordés aux migrants reposent sur l'évaluation par les décideurs des coûts et des avantages pour la population du pays d'accueil $^{8}$. II reconnaît avec prudence le poids moral des droits de l'homme, mais soutient que les raisonnements axés sur ces

5 D. Bell, «Justice for Migrant Workers? Foreign Domestic Workers in Hong Kong and Singapore » in Sor-hoon Tan (dir.), Challenging Citizenship: Group Membership and Cultural Identity in a Global Age, Surrey and Burlington, Ashgate 2005.

$6 \mathrm{GCIM}$, Migration in an Interconnected World ( $\left.n^{\circ} 24\right)$.

7 R. Pevnick, Immigration and the Constraints of Justice, CUP, 2011, p. 179.

8 Ruhs, Price of Rights, ( $\left.n^{\circ} 54\right), 13$. 
droits ne tiennent pas souvent compte des avantages économiques pour les migrants, et principalement de l'intérêt des futurs migrants qui cherchent à accéder au marché du travail des pays à revenu élevé. La reconnaissance d'un large éventail de droits et/ou d'une égalité des droits (comme citoyens) en faveur des migrants est susceptible d'avoir pour "prix », ou pour contrepartie, une politique d'admission plus restrictive vis-à-vis des futurs migrants.

II est cependant probable que cette argumentation soit utilisée de manière détournée pour justifier la privation de la quasi-totalité des droits ayant un coût éventuel. Le compromis entre "nombre de migrants et droits des moigrants" peut engendrer des dilemnes normatifs pour des États démocratiques libéraux attachés aux principes fondamentaux des droits de la personne et à l'égalité. Ruhs a tenté de préciser que si «certains droits génèrent des coûts", cela "ne signifie pas qu'il soit justifié moralement de cautionner ou de préconiser de telles restrictions ${ }^{9}$ ». Pourtant, le " prix» de ces droits semble impliquer que certains objectifs réglementaires tels que l'efficacité économique soient privilégiés par rapport à d'autres. Une telle optique peut affecter la légitimité morale, le poids des droits de l'homme et des principes d'universalité, d'inaliénabilité et d'indivisibilité. Castles s'est interrogé sur le fait de savoir s'il est acceptable d'échanger les droits des travailleurs contre des gains économiques ${ }^{10}$.

Les exemples de politiques de migration économique qui limitent simultanément les droits des migrants et le nombre de migrants ne sont pas rares dans de nombreux États libéraux comme le Royaume-Uni. L'analyse de Ruhs s'est largement limitée à lıexamen des "droits sur papier». Bien qu'il reconnaisse qu'« en théorie, les migrants peuvent se voir refuser certains droits consacrés légalement ${ }^{11}$ ", l'enquête de Ruhs ne va pas jusqu'à un examen des « droits dans la pratique ». Notamment, son enquête n'a pas prêté suffisamment attention aux effets des statuts juridiques précaires de ces migrants sur la mise en œuvre concrète de leurs droits en matière d'emploi.

\section{II - LA REFONTE DES TMWP}

Diverses propositions ont été faites en vue de la refonte des TMWP contemporains. Ces propositions, qui sont examinées ici, reposent sur un point commun : I'octroi de certains droits aux migrants dans le cadre des TMWP, tout en restreignant une série d'autres droits. Cependant, les propositions diffèrent par leurs prémisses.

L'aptitude de Ruhs à tolérer certaines restrictions des droits en échange de l'accès des migrants au marché du travail de l'État hôte est fondée sur l'octroi d'un ensemble de droits " essentiels " aux migrants dans le cadre des TMWP ${ }^{12}$. Son argumentation repose sur l'idée que les droits civils et politiques fondamentaux des migrants (à l'exception du droit de vote) ne doivent pas être restreints en vertu d'un quelconque régime juridique. D'autres droits peuvent en revanche être restreints si l'on peut prouver qu'ils engendrent des coûts

9 Ibid. 4.

10 Castles, « Guestworkers in Europe: A Resurrection? » ( $\left.n^{\circ} 3\right)$ p.749.

11 Ruhs, Price of Rights, ( $\left.{ }^{\circ} 54\right), 12$.

12 Ibid. 
nets pour les États hôtes. Il soutient qu'il faut limiter les restrictions à celles qui existent déjà dans les États démocratiques à revenu élevé, à savoir par exemple le droit au libre choix de l'emploi, le droit au regroupement familial et le droit à une résidence permanente.

Pevnick propose également de reconnaître un ensemble de droits fondamentaux, " essentiels» dans le cadre d'un régime de travailleurs invités éthiquement acceptable, qui permettrait aux migrants de travailler pour différents employeurs et de protéger leurs droits fondamentaux en matière d'emploi. En ce qui concerne les droits qui peuvent être exclus ou restreints, il vise l'accès à la citoyenneté, à la sécurité sociale, au regroupement familial $»^{13}$.

La distinction faite par Ruhs et Pevnick entre les droits essentiels et droits nonessentiels dans le cadre des TMWP risque de renforcer la démarcation entre les droits civils et politiques d'un côté et les droits sociaux et économiques de l'autre, selon une hiérarchie donnant la priorité aux premiers. De plus, toute rationalisation visant à faire la distinction entre droits essentiels et droits non-essentiels doit dépasser les arguments fondés sur le «coût» de ces droits. Ces propositions semblent reposer sur une vision instrumentale selon laquelle les droits accordés aux migrants sont déterminés par des considérations coûts-avantages qui « dépendent de leurs impacts sur la population locale dans le pays d'accueil ${ }^{14}$ ». De tels arguments ont peu d'attrait pour les défenseurs des droits de l'homme et de l'engagement de l'État en faveur de l'universalité, l'inaliénabilité et l'indivisibilité des droits de l'homme.

Reconnaissant les contraintes morales qui pèsent sur les démocraties libérales lorsqu'il s'agit d'opérer une distinction entre les citoyens et les non-citoyens pour attribuer des droits, Carens a adopté une approche normative plus substantielle qui identifie les circonstances dans lesquelles les États libéraux peuvent légitimement accepter des travailleurs migrants de manière temporaire et imposer certaines restrictions des droits. II admet que les restrictions imposées en petit nombre sur les droits sociaux peuvent être justifiées par le caractère temporaire du séjour des migrants dans le pays d'accueil.

Pour Carens, l'admission de travailleurs migrants temporaires assortie de restrictions explicites à leur droit de séjour dans le pays d'accueil serait moralement acceptable à condition que ces travailleurs ne soient effectivement présents que pour une courte période. Pour les personnes admises à travailler avec un visa temporaire « qui n'ont d'autre droit de résidence que leur présence dans le pays ${ }^{15}$ ", il serait raisonnable de s'attendre à ce qu'elles quittent le pays d'accueil après un an ou deux, à l'expiration de leur visa. Cependant, pour Carens, « il n'est pas acceptable de maintenir les personnes dans un statut temporaire qui serait constamment renouvelé, si bien qu'en réalité, elles resteraient longtemps, voire définitivement, dans une situation de vulnérabilité et avec des droits restreints ${ }^{16} »$.

13 Pevnick, Immigration and the Constraints of Justice (n 8) 178

14 Ruhs, Price of Rights, (n 5) 13.

15 J. H. Carens, "Live-in Domestics, Seasonal Workers, and Others Hard to Locate on the Map of Democracy », Journal of Political Philosophy, vol. 16, 2008, p. 422.

16 J. Carens, "Who Belongs? Immigration, Democarcy, and Citizenship» in O. Schmidtke and S. Ozcurumez (dir), Of States, Rights, and Social Closure, Palgrave Macmillan 2007, p. 17. 
Plutôt que de promouvoir l'extension des droits aux migrants temporaires, Ottonelli et Torresi exigent la mise en place d'options, de renoncements possibles à tous les stades du projet de migration. Les mesures peuvent comprendre le fait de donner aux migrants la possibilité de changer d'emploi et de retourner dans leur pays d'origine quand ils le souhaitent, de permettre le transfert des prestations de sécurité sociale dans le pays d'origine, de permettre des visites fréquentes et des interactions avec le pays d'origine ${ }^{17}$.

\section{III - «TOWARDS EXIT AND VOICE $»^{18}$}

Notre propos est de reprendre la proposition Hirschman "Exit, Voice, and Loyalty »"19 pour l'appliquer aux travailleurs migrants mécontents qui entendent rompre la relation de travail pour chercher de meilleures conditions de travail ou qui recourrent à des mécanismes $d^{\prime}$ « expression » collective tels que le syndicalisme ou la négociation collective ${ }^{20}$. Exit, Voice sont généralement considérées comme des compromis puisque l'absence d'« expression " collective peut augmenter la probabilité d'une rupture de la relation et vice versa, comme le montre le lien établi par Freeman entre le syndicalisme et les taux de démission ${ }^{21 .}$

Dans certaines situations, les travailleurs sont privés de la possibilité de recourir à des mécanismes de rupture et d'expression. Selon Hirschman, les coûts initiaux d'entrée élevés, les pénalités sévères et les sanctions en cas de rupture peuvent diminuer voire supprimer l'importance du compromis Exit, Voice. Hirschman identifie ces frais d'entrée et ces pénalités à des dispositifs permettant aux organisations de générer ou de renforcer la loyauté des consommateurs ou des salariés. En d'autres termes, la loyauté perturbe le fonctionnement de Exit, Voice en incitant les consommateurs ou les salariés à rester au sein d'une organisation ${ }^{22}$.

Les coûts d'entrée peuvent inclure le temps, l'argent, les ressources et l'énergie investis par le migrant, sa famille et sa communauté pour se lancer dans le projet de migration. Dans le même temps, les pénalités en cas de rupture de la relation peuvent être lourdes. Si un migrant bénéficiaire d'un TMWP quitte son poste de travail, il pourrait en subir des conséquences négatives comme la perte de l'autorisation légale de séjour dans le pays d'accueil, voire courir le risque d'une expulsion. D'autres sanctions possibles peuvent consister en une obligation de remboursement de dettes importantes, ou plus simplement résider dans la honte associée à un retour anticipé.

17 V. Ottonelli et T. Torresi, « Inclusivist Egalitarian Liberalism and Temporary Migration: A Dilemma », Journal of Political Philosophy», vol. 20, 2012, p. 220.

$18 \mathrm{Ndlr}$ : Si on devait traduire cette expression en langue française, nous pourrions proposer de la comprendre comme l'expression d'un choix entre liberté de rupture et droit d'expression collective. Néanmoins, nous préférons par souci de fidélité à l'intention de l'auteur reprendre telle quelle cette expression en langue anglaise.

19 A. O. Hirschman, Exit, Voice, and Loyalty, Cambridge MA, Harvard University Press, 1970.

20 R. B. Freeman and J. L. Medoff, What Do Unions Do?, New York, Basic Books, 1984.

21 R. B Freeman, "The Exit-Voice Tradeoff in the Labor Market: Unionism, Job Tenure, Quits, and Separations ", The Quarterly Journal of Economics, n 94, 1980, p. 643.

22 Hirschman $n^{\circ} 18$, p. 93. 
Plus les sanctions en cas de rupture sont lourdes, plus les mécanismes d'expression deviennent importants pour les travailleurs. II semblerait que le rattachement du statut juridique des migrants à un parrainage par un employeur dans le cadre de TMWP oblige ou contraint à la « loyauté » les travailleurs migrants ainsi immobilisés par des droits d'entrée importants et des pénalités de rupture prohibitives. L'utilisation du droit d'expression selon un canal individuel ou collectif, formel ou informel, comme les procédures judiciaires, la négociation collective ou les procédures amiables de résolution des litiges,est compromise dans de telles situations. Les migrants peuvent être réticents à exprimer un mécontentement ou une plainte contre leur parrain ou leur employeur lorsque la rupture n'est pas une possibilité viable.

L'élargissement des possibilités de cessation de la relation de travail des migrants est essentiel pour qu'ils puissent s'engager librement sur le marché du travail du pays de résidence et plus généralement pour faciliter leurs projets de vie, liés à la réalisation de leur projet migratoire. La possibilité de mettre un terme à une relation de travail et/ou à un projet migratoire défaillant pour certains travailleurs migrants et leurs familles peut engendrer de lourdes contraintes pratiques comme la nécessité de rembourser des dettes importantes contractées avant et pendant le voyage migratoire. Cela rend d'autant plus important le recours au droit d'expression, notamment en ce qui concerne les mécanismes de représentation et de mobilisation collective qui peuvent améliorer le pouvoir de négociation individuel d'un travailleur qui a peu ou pas de possibilité de rupture. L'accès à des mécanismes de représentation efficaces peut constituer un rempart contre l'exploitation par l'autonomisation collective et la mobilisation des travailleurs migrants dans le cadre des TMWP, comme pour leurs homologues bénéficiant de la résidence permanente et de la citoyenneté.

\section{IV - PROPOSITION DE RÉFORME BASÉE SUR EXIT AND VOICE}

\section{A - DÉTACHER LE STATUT DE RÉSIDENT DU PARRAINAGE PAR L'EMPLOYEUR}

L'élimination des contraintes légales sur la mobilité des migrants est essentielle à l'exercice de la liberté de rupture des migrants, et minimiserait le risque de voir des travailleurs liés de force à un employeur et à une relation de travail. Bien que les TMWP n'empêchent pas explicitement les migrants de changer d'employeur, leur autorisation légale de travailler et de résider dans le pays d'accueil est basée essentiellement sur un accord de parrainage avec un employeur ou un utilisateur de main-d'œuvre déterminé.

Ruhs soutient que la portabilité des autorisations de travail ou des visas entre différents employeurs pourrait être limitée de manière acceptable à une courte période (par exemple six mois), sinon cela réduirait considérablement la propension des employeurs locaux à recruter des travailleurs migrants s'ils sont libres de quitter l'employeur qui les a recrutés avant qu'une partie des coûts du recrutement n'ait été récupérée par ledit employeur ${ }^{23}$ ». 
Carens propose une période de trois mois maximum pour limiter la liberté des migrants de changer d'employeur, de sorte que les " coûts de recrutement " puissent être récupérés. Même avec une telle restriction, il devrait exister une clause de sauvegarde en cas de comportement abusif de la part de l'employeur durant cette période ${ }^{24}$.

Sans la liberté de rompre une relation de travail à tout moment, un migrant peut être soumis à des formes graves d'exploitation qui ne sont pas acceptables, même pour une " courte période ». S'il est vrai que Carens propose une clause de sauvegarde, il ne précise pas comment cela fonctionnerait en pratique. Une telle clause semble viser des situations de travail extrêmement abusives a posteriori, alors qu'il serait préférable d'empêcher que ne se crée cette source de vulnérabilité des migrants engendrée par des restrictions à l'immigration. Une réponse raisonnable à la nécessité des employeurs de récupérer les coûts de recrutement consisterait à réduire les frais de visa et les frais administratifs pour les employeurs et les travailleurs migrants (ce qui pourrait aussi réduire la dette des migrants).

Au cœur de notre proposition se trouve l'idée selon laquelle l'autorisation légale du migrant de travailler et de résider dans le pays d'accueil devrait être détachée du contrat de travail le liant à un parrain spécifique. Les migrants peuvent se voir délivrer une autorisation de travail transférable non liée au parrainage particulier d'un employeur, qui leur permette de changer d'employeur et de chercher un autre emploi pendant toute la durée de l'autorisation de séjour. Les intermédiaires du marché du travail et les ministères du travail peuvent jouer un rôle constructif en canalisant l'information et les ressources pour mettre en relation les migrants avec les employeurs des zones de pénurie identifiées avant, à leur arrivée, ainsi que pendant leur séjour dans le pays d'accueil.

De nouveaux types d'intermédiaires tels que les entreprises sociales identifiées par l'étude de Forde et MacKenzie ${ }^{25}$ peuvent jouer un rôle positif pour répondre aux besoins des travailleurs migrants tout au long de leur projet migratoire, par exemple, en facilitant la transition d'un emploi à un autre. Un cadre réglementaire solide concernant les obligations et les responsabilités en matière d'enregistrement et d'octroi de licences, de suivi et d'application peut contribuer à réduire les éventuelles pratiques abusives d'intermédiaires moins scrupuleux.

\section{B - Mobilité PROFESSIONNELLE ET SECTORIELLE ET « PÉNURIE DE COMPÉTENCES »}

Une des principales justifications des TMWP comme instruments d'une politique visant à remédier à des pénuries de compétences spécifiques a été utilisée pour justifier les restrictions sur le libre choix de l'emploi des migrants dans le cadre de ces programmes. Vues les contraintes pesant sur la liberté des migrants de changer d'employeur, les restrictions à la mobilité de la main-d'œuvre en ce qui concerne la profession et/ou le secteur peuvent être conçues de telle sorte que les travailleurs puissent parfois avoir des possibilités de rupture et d'expression. La proposition principale est que le choix de l'emploi des migrants

24 Carens, "Live-in Domestics », n 15, p. 433.

25 Ch. Forde et R. MacKenzie, "The Ethical Agendas of Employment Agencies Towards Migrant Workers in the UK: Deciphering the Codes », Journal of Business Ethics, vol. 97, 2010, p. 31. 
pourrait se limiter à une liste définie de professions et de secteurs connaissant une pénurie de main-d'œuvre attestée sur le marché du travail local. Les autorisations de travail des migrants leur permettraient de choisir entre les professions de cette liste et d'opérer des transferts.

Un outil politique rigoureux permettant d'identifier les véritables pénuries de maind'œuvre consisterait à recourir à un groupe d'experts indépendants pour compiler et mettre régulièrement à jour une liste de professions connaissant des pénuries, avec le concours des parties prenantes, y compris les migrants et leurs organisations. Par exemple, le Migration Advisory Committee (MAC) au Royaume-Uni est un organe d'experts indépendants qui effectue des analyses régulières des pénuries de main-d'œuvre qualifiée et conseille le gouvernement sur une série de réponses politiques appropriées, qui vont au-delà de la migration des travailleurs. Le MAC s'appuie à la fois sur des données « descendantes » du marché du travail et sur des données « ascendantes " provenant d'employeurs, de groupes d'affaires, de syndicats et de ministères. Plutôt que de simplement se fier à l'affirmation de l'employeur selon lequel il existe des "pénuries", cette approche pourrait prendre en compte des facteurs plus larges du marché du travail et de l'économie, ainsi que les intérêts de divers acteurs du marché du travail.

\section{C - Protections de L'EMPLOI ET ORgANisation Collective}

Dans les États démocratiques et libéraux, les lois sur la protection de l'emploi s'appliquent généralement de la même manière aux migrants temporaires (ayant un statut de résident légal) qu'aux résidents permanents et aux citoyens ${ }^{26}$. La protection efficace des droits du travail des migrants exige de distinguer leur statut de migrant de l'application des lois sur l'emploi. Un modèle de TMWP qui n'associe pas l'autorisation d'emploi et de résidence du migrant à un parrainage obligatoire de l'employeur atténuerait les craintes de représailles si les migrants cherchaient à porter plainte contre leur employeur en cas de violation de leurs droits.

L'application de contrôles à l'immigration est aussi une source importante d'obstacles pratiques à l'accès des migrants à la protection de l'emploi. La fonction de protection des travailleurs, inhérente à l'inspection du travail, peut être sérieusement compromise lorsque les inspecteurs sont aussi censés représenter les autorités de l'immigration. L'application des droits des migrants dans le travail exige que les inspections du travail s'acquittent de leur mandat sans compromis. Un "pare-feu légal ${ }^{27}$ " devrait être mis en place entre l'application de la loi sur l'immigration et la protection des droits du travail des migrants pour permettre aux migrants ayant des statuts précaires de pouvoir revendiquer ces droits.

Fondamentalement, les syndicats peuvent jouer un rôle essentiel dans l'organisation des migrants dans le cadre des TMWP afin d'élargir leurs possibilités d'expression sur le lieu de travail, en particulier lorsque leurs possibilités de rupture sont limitées. Les migrants doivent pouvoir adhérer librement aux syndicats de leur choix, solliciter l'assistance des syndicats pour faire valoir leurs droits et s'organiser collectivement pour améliorer leurs salaires et leurs conditions de travail sans craindre de mettre en péril leur statut juridique.

26 Carens, 'Live-in Domestics', n 15, p. 425.

27 J. Carens, «The Rights of Irregular Migrants », Ethics and International Affairs, vol. 22, 2008, p. 167. 
Cela met à nouveau en évidence la nécessité de ne pas lier l'autorisation d'entrée et de séjour des migrants dans le pays d'accueil au parrainage d'un employeur déterminé. Les syndicats peuvent en outre être un moyen de surmonter les divisions de la main-d'œuvre en favorisant la solidarité entre les migrants et les travailleurs résidents, les migrants plus établis et les nouveaux migrants, ainsi que les différents groupes ethniques et raciaux. II peut y avoir un alignement des intérêts entre les travailleurs migrants et les travailleurs résidents dans la lutte contre les relations de travail abusives que la mobilisation collective et l'autonomisation peuvent aider à atteindre.

Il est nécessaire également que les syndicats innovent dans l'organisation et la mobilisation de la main-d'œuvre migrante "temporaire", par exemple en nouant des alliances avec les communautés de migrants et les organisations de la société civile. Il existe également un potentiel d'organisation transnationale entre les syndicats des pays d'accueil et des États d'origine, via la participation des organisations syndicales internationales. Cependant, la tentative ratée de l'Association européenne des travailleurs migrants de devenir une structure organisationnelle transnationale pour les travailleurs migrants détachés dans le secteur de la construction révèle les limites politiques de la solidarité transfrontalière entre les mouvements ouvriers nationau ${ }^{28}$.

\section{D - PRotection sociale}

La possibilité d'accéder aux droits sociaux dans le pays d'accueil, qu'il s'agisse des prestations de chômage et maladie ou de l'éducation et des services de logement, peut être plus faciles pour les migrants sous TMWP et pour les membres de leur famille. Cependant, la restriction de l'accès des migrants à la protection sociale est souvent défendue pour maximiser la «contribution nette fiscale des migrants » et en minimiser le «coût» pour le pays d'accueil et les citoyens de l'extension des services publics et des prestations sociales aux migrants ${ }^{29}$.

Cependant, le principe de l'inclusion dans les États libéraux exige au moins que ces personnes soient admises comme membres temporaires du personnel de l'entreprise et de la société qui les accueille (travail et vie aux côtés des résidents permanents et des citoyens, travail rémunéré, obéissance aux lois du pays d'accueil); ils ne devraient pas être exclus des avantages sociaux pendant leur séjour. A minima, l'accès aux avantages sociaux " universels » comme les services de santé publique et l'éducation dans les pays d'accueil devrait être possible en droit et en pratique pour les migrants et les membres de leur famille dans le cadre des TMWP.

La distinction nuancée de Carens entre les différents types de prestations sociales selon la nature et le but est plus convaincante. Sur la base du principe de réciprocité, il soutient que les migrants temporaires devraient disposer des mêmes droits ou avoir droit au remboursement des contributions à des programmes liés à leur participation au marché du travail. Tandis que les travailleurs migrants et leurs familles ne peuvent pas bénéficier de ces avantages sociaux en vertu des lois nationales applicables, l'article 27 (2) de la

28 I. Greer, Z. Ciupijus et N. Lillie, «The European Migrant Workers Union and the barriers to transnational industrial citizenship », European Journal of Industrial Relations, vol. 19, 2013, p. 5.

29 Ruhs, Price of Rights, ( $\left.{ }^{\circ} 5\right), 47$. 
Convention internationale sur la protection des droits de tous les travailleurs migrants et des membres de leur famille (ICPRMW) encourage l'État à rembourser «le montant des cotisations versées au titre de cette prestation, sur la base du traitement accordé aux nationaux qui se trouvent dans des circonstances similaires $»$.

Une proposition réalisable consisterait à ce qu'une partie des impôts sur le revenu et des prestations de sécurité sociale perçus par l'État auprès des travailleurs migrants et des membres de la famille soit versée dans un fonds spécial pour financer l'accès aux prestations sociales pendant leur séjour dans le pays d'accueil. Pour faciliter leur rupture avec le projet migratoire et leur retour vers le pays d'origine, des accords bilatéraux entre les pays d'accueil et d'origine pour garantir la portabilité de ces fonds constitueraient des protections vitales pour les migrants et leurs familles contre les risques de dénuement pendant leur séjour dans le pays d'accueil et lors de leur retour dans le pays d'origine.

Les revendications des migrants temporaires vis-à-vis des programmes sociaux fournis par l'État au bénéfice des citoyens peuvent être restreintes de façon plausible lorsque les programmes visent une redistribution, telle que les aides au revenu et les logements sociaux. Cependant, les revendications des migrants vis-à-vis de ces avantages devraient se renforcer au cours du séjour dans le pays d'accueil. Une période de résidence raisonnable (par exemple 1 à 2 ans) entre le moment où les migrants arrivent dans le pays d'accueil et le moment où ils peuvent prétendre à des prestations sociales non contributives serait acceptable et conforme à la norme internationale sur le droit à la sécurité sociale ${ }^{30}$.

\section{E - DROIT À L'ACCOMPAGNEMENT OU AU REGROUPEMENT FAMILIAL}

Dans le cadre des TMWP, il existe généralement des restrictions sur l'accompagnement ou le regroupement familial. Outre les interdictions explicites, le migrant peut être tenu de démontrer qu'il dispose d'un niveau de revenu et de ressources suffisant pour subvenir aux besoins des membres de sa famille ou prétendre à une demande de regroupement familial après une période de résidence dans le pays d'accueil.

Toute restriction au regroupement ou à l'accompagnement familial doit prendre en compte le poids du droit de mener une vie familiale inscrit dans pléthore d'instruments internationaux, régionaux et nationaux relatifs aux droits de l'homme. Dans le contexte spécifique des migrants, en vertu de l'article 44.2 de l'ICPRMW, «Les États parties prennent les mesures qu'ils jugent appropriées et qui relèvent de leur compétence pour faciliter la vie commune des travailleurs migrants avec leur conjoint ou avec les personnes ayant avec eux des relations qui, en vertu de la loi applicable, produisent des effets équivalant au mariage, ainsi qu'avec leurs enfants à charge mineurs et célibataires ».

30 CESCR, Observation générale $n^{\circ}$ 19, Le droit à la sécurité sociale (Art 9) (adopté le 23 novembre 2007) U.N. Doc. E/C.12/GC/19 (2008). 
Comme le note Carens, «les États démocratiques et libéraux ont du mal à justifier l'annulation de ce droit uniquement par souci de préserver des intérêts purement économiques ${ }^{31}$ ». Si une telle restriction devait être imposée dans le cadre d'un TMWP, la période de restriction devrait être aussi limitée que possible, par exemple, les premiers mois du projet migratoire avec la possibilité de rentrer chez soi pour rendre visite à ses proches.

Selon le modèle proposé, le droit des migrants à l'accompagnement ou au regroupement familial ne serait pas subordonné à l'existence d'une relation de travail avec un employeur déterminé. Le risque de mettre un terme au programme de migration est plus fort si les migrants se retrouvent piégés dans des relations de travail abusives et/ou dans des projets migratoires défaillants dans le pays d'accueil. En outre, les membres de la famille doivent avoir le droit de travailler sur le marché du travail du pays de résidence et avoir accès aux droits sociaux susmentionnés.

\section{F - DROIT D'OBTENIR UN STATUT DE RÉSIDENT}

Deux options différentes basées sur le principe selon lequel, dans le cadre des TMWP, les migrants devraient avoir un statut de résident légal clair et sécurisé tout au long de leur projet migratoire peuvent être proposées.

La première, c'est que les TMWP devraient être une voie vers la résidence permanente, intégrée dès le début parmi les conditions d'admission au séjour. Si le TMWP permet le renouvellement continu des visas et des permis de travail des migrants, ceux-ci devraient pouvoir demander la résidence permanente après une période minimale d'emploi et de résidence dans le pays d'accueil. Il est important de noter que cette transition vers le statut de résident permanent ne devrait pas nécessiter le parrainage et/ou le soutien d'un employeur déterminé. La difficulté sera de savoir quand les migrants temporaires acquièrent le «droit» au statut de résident permanent. L'Union européenne ${ }^{32}$ accorde le statut de résident permanent aux migrants résidant légalement dans un État membre depuis cinq ans.

La deuxième possibilité consiste à rendre les TMWP véritablement temporaires en limitant explicitement le renouvellement des autorisations de travail et des visas. Comme l'affirme Carens, " l'admission temporaire de travailleurs est moralement admissible, mais seulement si la durée de leur séjour est vraiment limitée... les États démocratiques ne peuvent pas maintenir indéfiniment les gens dans un statut «temporaire " ${ }^{33}$. Pendant le séjour temporaire du migrant dans le pays d'accueil, son statut juridique ne devrait pas être compromis ni relever du pouvoir discrétionnaire de l'employeur quant au renouvellement ou à la cessation de son parrainage. Si les migrants dans le cadre du TMWP ne peuvent pas bénéficier de possibilités indépendantes de résidence permanente (qui ne dépendent pas du parrainage d'un employeur), limiter le renouvellement des autorisations de travail et des visas des migrants constituerait une option de second choix.

31 Ibid.

32 Directive 2003/109/CE du Conseil du 25 novembre 2003 relative au statut des ressortissants de pays tiers résidents de longue durée.

33 J. Carens, The Ethics of Immigration, Oxford, OUP, 2013, p. 113. 
L'argument principal réside dans le caractère indéfendable de l'idée de maintenir les migrants dans une situation de statut légal précaire sur le long terme, ce qui renforcerait leur dépendance vis-à-vis de l'employeur et donnerait une extrême incertitude à leur projet migratoire. On peut également tenir compte du fait que certains migrants entreprennent des « projets de migration temporaire » sans intention de s'installer dans le pays d'accueil seulement pour obtenir les revenus et l'expérience nécessaires pour mener à bien leur vie à long terme, une fois rentrés dans leur pays ${ }^{34}$. Néanmoins, plus ce groupe de résidents "temporaires» reste longtemps, plus ils sont susceptibles de développer des formes de liens affectifs, psychologiques, sociaux, culturels et économiques avec la société d'accueil. Dans les États démocratiques et libéraux, il y a une forte justification morale au fait que ces migrants soient incorporés comme membres à part entière du pays d'accueil sur la base de liens qui se renforcent avec le temps.

\section{G - LÉGISLATION, ADMINISTRATION ET MISE EN đEUVRE}

Enfin, on peut envisager l'idée qu'un type de réglementation et dradministration clair, transparent et responsable étayant les TMWP qui contribuerait à remédier à certains défauts de fond et de forme qui précarisent la situation des migrants. Des règles d'immigration complexes, incohérentes et souvent bricolées ainsi que des procédures opaques et inefficaces peuvent augmenter la probabilité de violations involontaires des droits autant par les employeurs que par les migrants. Comme le dit Anderson, «paradoxalement dans un système de contrôle bureaucratique et inévitablement complexe, l'État peut perdre le contrôle de la migration et provoquer ainsi une augmentation du nombre de personnes en situation de séjour irrégulier de personnes travaillant en violation du droit, et d'immigrés clandestins ${ }^{35} »$.

Disposer d'informations fiables sur le TMWP, sur les conditions de travail et de vie, sur les lois et protections en matière d'emploi et les règles d'immigration est cruciale pour aider les migrants à faire des choix informés et réfléchis sur leur projet migratoire. Dans la pratique, les migrants recherchent ces informations auprès des employeurs, des intermédiaires du marché du travail et dans leurs réseaux, y compris les amis, les familles et les autres migrants. L'information donnée est souvent délibérément ou involontairement inexacte. Les migrants peuvent également devenir dépendants d'une ou deux de ces sources d'information. II est par conséquent réellement nécessaire de disposer de mécanismes permettant d'offrir des informations précises et à jour (dans leur propre langue), auxquelles les migrants puissent accéder pour les aider à prendre des décisions plus éclairées concernant leur projet migratoire et faciliter leur faculté de rupture de la relation de travail et d'expression collective

34 Ottonelli et Torresi, $\mathrm{n}^{\circ} 17$.

35 Anderson (n 3) p. 311. 


\section{Conclusion}

Lors de la conception des TMWP, les décideurs des États démocratiques et libéraux doivent s'attaquer à un ensemble de questions normatives et éthiques. L'opinion dominante qui oppose au nombre de migrants les droits des migrants reflète en grande partie une approche utilitariste fondée sur des considérations de comparaison des coûts par rapport aux avantages pour le pays d'accueil. Les propositions de révision des TMWP consistent à accorder aux migrants un petit ensemble de droits "essentiels», tout en restreignant d'autres droits en plus grand nombre. Cependant, de telles propositions requièrent une norme substantielle qui identifie et justifie les droits «fondamentaux» de ceux qui ne le sont pas

Le recours à des mécanismes de rupture et d'expression collective durant toute la relation de travail des migrants et de leur projet migratoire suppose d'intégrer les propositions de réforme ici présentées. Ces propositions cherchent en outre à intégrer les " aspirations et les projets des migrants eux-mêmes ${ }^{36}$ " dans les TMWP. Compte tenu des puissants facteurs d'incitation et d'attraction qui alimentent les migrations mondiales de main-d'œuvre, il est peu probable que liutilisation des TMWP cesse dans un proche avenir. C'est pourquoi, nous avons préféré envisager de réformer les TMWP, plutôt que de plaider pour leur élimination immédiate. Plutôt que de présenter un plan pour un TMWP permettant de gagner sur trois tableaux, il est permis d'espérer que les propositions susmentionnées puissent attirer l'attention des décideurs des États démocratiques et libéraux sur la légitimité normative et la faisabilité pratique de protéger les migrants contre une extrême précarité sur le lieu de travail et au-delà.

36 Ottonelli et Torresi, $n^{\circ} 17$, p. 208.

\section{MIMI ZOU}

Chercheur à l'Université d'Oxford. Thèmes de recherche : Droit du travail, migration internationale, droit commercial, droit chinois.

Publications:

M. Zou, "The Regulatory Challenges of "Uberization" in China: How to Classify Ride-Hailing Drivers?'", International Journal of Comparative Labour Law and Industrial Relations, 2017, n 33, p. 269.

$\sim$ M. Zou, « The Legal Construction of Hyper-dependence and Hyper-Precarity in Migrant Work Relations», International Journal of Comparative Labour Law and Industrial Relations, 2015, $n^{\circ} 31$, p. 141. 